\title{
Pressure-sensitive reaction yield of the TePixD blue-light sensor protein
}

\section{$\operatorname{AUTHOR}(\mathrm{S}):$}

Kuroi, Kunisato; Okajima, Koji; Ikeuchi, Masahiko; Tokutomi, Satoru; Kamiyama, Tadashi; Terazima, Masahide

\section{CITATION:}

Kuroi, Kunisato ... [et al]. Pressure-sensitive reaction yield of the TePixD blue-light sensor protein. The Journal of Physical Chemistry B 2015, 119(7): 2897-2907

\section{ISSUE DATE:}

2015-02-19

URL:

http://hdl.handle.net/2433/218346

\section{RIGHT:}

This document is the Accepted Manuscript version of a Published Work that appeared in final form in 'Journal of Physical Chemistry B', copyright @ American Chemical Society after peer review and technical editing by the publisher To access the final edited and published work see http://doi.org/10.1021/jp511946u.; この論文は出版社版でありませ ん。引用の際には出版社版をご確認ご利用ください。; This is not the published version. Please cite only the published version. 


\title{
Pressure-sensitive reaction yield of the TePixD blue-light sensor protein
}

Kunisato Kuroi, ${ }^{\dagger}$ Koji Okajima, ${ }^{\ddagger}{ }^{\prime}$ Masahiko Ikeuchi, ${ }^{\ddagger}$ Satoru Tokutomi, ${ }^{\S}$ Tadashi Kamiyama, ${ }^{\circledR}$ and Masahide Terazima*,†

†Department of Chemistry, Graduate School of Science, Kyoto University, Kyoto 606-8502, Japan

"Department of Life Sciences (Biology), Graduate School of Arts and Sciences, The University of Tokyo, Meguro, Tokyo 153-8902, Japan

${ }^{\S}$ Department of Biological Science, Graduate School of Science, Osaka Prefecture University, Sakai, Osaka 599-8531, Japan

"Department of Chemistry, School of Science and Engineering, Kinki University, Higashi-Osaka, Osaka 577-8502, Japan

\begin{abstract}
The effect of pressure on the dissociation reaction of the TePixD decamer was investigated by high-pressure transient grating (TG). The TG signal intensity representing the dissociation reaction of the TePixD decamer significantly decreased by applying a relatively small pressure. On the other hand, the reaction rate increased with increasing pressure. The equilibrium between the pentamer and the decamer was investigated by high-pressure dynamic light scattering. The results indicated that the
\end{abstract}


fraction of the decamer slightly increased in the high-pressure region. From these measurements, it was concluded that the pressure-dependent signal intensity originated from the decrease of the quantum yield of the dissociation reaction of the decamer, indicating that this reaction efficiency is very sensitive to pressure. Using densimetry at high pressures, the compressibility was found to be pressure dependent even in a relatively low pressure range. We attributed the origin of the pressure-sensitive reaction yield to the decrease of compressibility at high pressure. Because the compressibility is related to the volume fluctuation, this observation suggests that the driving force for this reaction is fluctuation of the protein. The relationship between the cavities at the interfaces of the monomer units and the reactivity is also discussed. 


\section{INTRODUCTION}

In general, proteins possess relatively large conformational flexibility. Because the protein conformation has to change during a reaction, this flexibility should be an essential factor for the reaction, and, consequently, for the biological function. Frequently, such flexibility originates from imperfect packing of the residues or cavities inside the protein structure, and this is represented by the volume properties of the protein. ${ }^{1,2}$ At room temperature in solution, the flexibility leads to conformation fluctuation of the protein. Hence, the relationship between the reactivity and the fluctuation (or the volume properties) is important to understand the protein reaction mechanism. In this respect, the pressure, which is closely related to the volume properties, can be a probe for the volume properties. In fact, pressure effects on protein reactions have been studied for several decades. ${ }^{3}$ The pressure dependence of the equilibrium constant provides information about the reaction volume, and the pressure dependence of the reaction kinetics provides information about the activation volume. For example, pressure effects on enzymatic reactions have been reported, and show that the reaction rate depends on the pressure. ${ }^{4-8}$ From the pressure dependence, the activation volume (i.e., the volume change at the transition state) was determined. In most cases, the enzymatic reactions were deactivated by high pressure (200-300 MPa), which was explained by pressure-induced structural changes. Pressure effects on the folding reactions of proteins provide information about the folding transition state, and suggest that proteins swell to a molten-globule state. ${ }^{9-12}$ The dissociation and binding processes of small molecules $\left(\mathrm{NO}, \mathrm{O}_{2}\right.$, and $\left.\mathrm{CO}\right)$ to/from hemeproteins have been examined by flash photolysis techniques. ${ }^{13-15}$ These studies clearly showed that pressure effects on the reaction kinetics and the equilibrium constants are useful to elucidate the 
reaction. However, although the pressure effect on the reactivity of protein reactions is important to understand the reaction, it has not been studied so far.

In this paper, we report, for the first time, a very pressure sensitive protein reaction yield of a blue light sensor protein, TePixD in a relatively small pressure range, and propose a mechanism related with the fluctuation of the protein. Photoreceptor proteins are important proteins, and are essential for most organisms from bacteria to higher plants and animals for sensing external light stimuli. ${ }^{16}$ These photoreceptors detect a photon by their covalently or non-covalently bound chromophores, and transmit the light information. TePixD is a BLUF (sensor of blue light using the flavin adenine dinucleotide) protein that exists in the thermophilic cyanobacterium Thermosynechococcus elongatus BP-1. TePixD forms a decamer structure. ${ }^{17}$ The $\mathrm{UV} / \mathrm{vis}$ absorption change associated with the photoreaction of TePixD is typical of BLUF proteins: ${ }^{18-21}$ a spectral red shift of $\sim 10 \mathrm{~nm}$ occurs upon photoexcitation within a nanosecond and recovers to the dark state in $\sim 5 \mathrm{~s}$. Our previous studies using the transient grating (TG) method revealed the spectrally silent reaction dynamics of TePixD. ${ }^{22,23}$ According to these studies, TePixD is in equilibrium between two oligomeric forms in the dark state: the decamer and the pentamer. Indeed, previous study showed that it is the mixture of the decamer and the pentamer within a concentration range of $50 \mu \mathrm{M}-200 \mu \mathrm{M}$ at the atmospheric pressure. Upon photoexcitation, it produces the first intermediate $\mathrm{I}_{1}$, which is characterized by a red-shifted absorption spectrum, and then undergoes volume expansion to produce the second intermediate $\mathrm{I}_{2}$. Although both the pentamer and decamer give these intermediates, only the decamer exhibits further reaction: it dissociates into two pentamers with an accompanied change in its diffusion coefficient (Figure 1). 

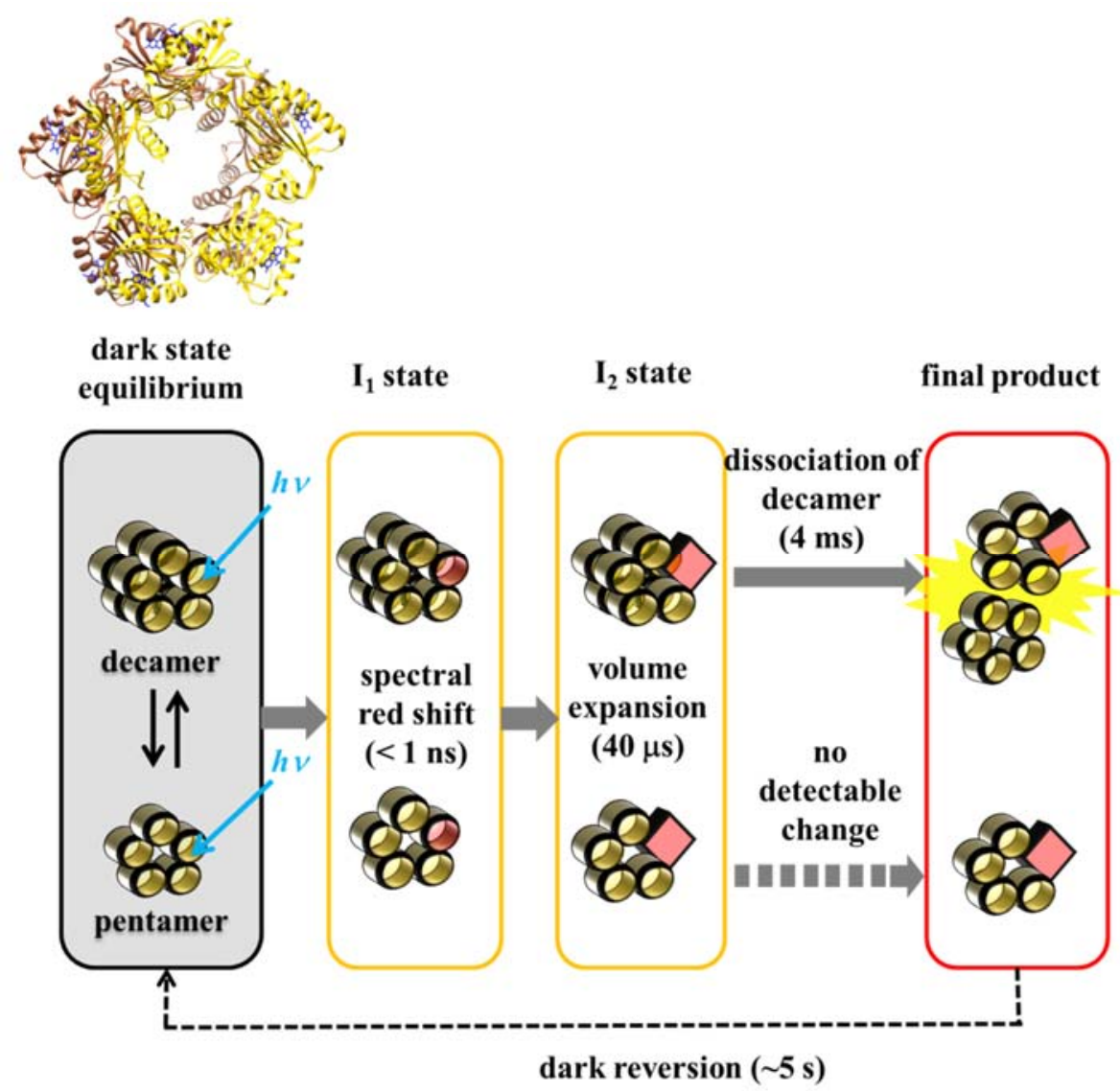

Figure 1. (Upper) Crystal structure of the TePixD decamer. The two different pentamer rings are colored yellow and brown, and the flavin adenine dinucleotide (chromophore) is shown in blue. (Lower) Schematic illustration of the photoreaction of TePixD. Yellow circles represent the TePixD monomer and red shows that the monomer unit is in the spectrally red-shifted state. The square shows that the excited monomer unit undergoes conformational change accompanied with volume expansion.

Furthermore, we discovered previously that the one monomer excitation and two monomer excitation lead different reactions by changing the light intensity. It has also 
been shown that transient enhancement of the fluctuation occurs during the reaction by measuring the compressibility in the time domain, ${ }^{24}$ that is, the fluctuations of both $\mathrm{I}_{1}$ and $I_{2}$ intermediates are larger than fluctuations in the ground state. According to this study, the pressure dependence of the quantum yield for the spectral shift reaction was small ( 20\% decrease at $200 \mathrm{MPa})$ and the quantum yield for the volume expansion process from $\mathrm{I}_{1}$ to $\mathrm{I}_{2}$ was also insensitive to pressure.

In this study, we investigated the effect of pressure on the dissociation reaction of the TePixD decamer by the TG method, as well as by dynamic light scattering (DLS) and densimetry at various pressures. We surprisingly found that the quantum yield for the dissociation reaction was very sensitive to pressure $(\sim 90 \%$ decrease at $100 \mathrm{MPa})$. We explain this phenomenon in terms of the pressure-dependent fluctuation of TePixD. As far as we know, this is the first report of the effect of pressure on the reaction quantum yield of a protein.

\section{EXPERIMENTAL METHODS}

2.1. Sample Preparation. TePixD was expressed using a pET28a vector transformed into Escherichia coli BL21 (DE3) and purified by nickel affinity column chromatography, as reported previously. ${ }^{25}$ In all of the measurements, the sample was prepared by dissolving in HEPES buffer (20 mM HEPES-NaOH (pH 7.5), $500 \mathrm{mM}$ $\mathrm{NaCl}$ ) and filtering through a centrifugal filter (Durapore PVDF $0.2 \mu \mathrm{m}$, Millipore) to remove dust particles before use. The concentration of TePixD was determined by UVVis absorption measurement using the extinction coefficient of flavin adenine dinucleotide at $450 \mathrm{~nm}\left(\varepsilon=11,300 \mathrm{M}^{-1} \mathrm{~cm}^{-1}\right)$. In most cases, the sample concentration was $\sim 330 \mu \mathrm{M}$.

2.2. High-Pressure Equipment for TG and DLS Measurements. Details of the 
high-pressure cell used for the TG measurement in this study were described elsewhere. ${ }^{26}$ The pressure resistance of this cell was up to $500 \mathrm{MPa}$. Although the TG signal intensity is generally very sensitive to any external perturbations, such as small movement of the cell or vibration of the sample, it was confirmed that the TG signal intensity was stable and reproducible for changing pressure and the sample exchange operation.

In the DLS measurements, we used a high-pressure cell PCI-400-DLS (Syn Corporation, Japan) that was specially designed to measure the light scattering. It had a cubic shape and three windows. Two window ports were mounted on opposite sides of the cell to introduce a laser beam, and the third large window was perpendicular to the optical axis to transmit the scattering light. The large window allowed the maximum scattering angle to be $27^{\circ}$. The pressure resistance of this cell was up to $400 \mathrm{MPa}$.

For both pressure apparatus, the temperature of the cell was controlled by circulating temperature-controlled water (mostly at $295 \mathrm{~K}$ in the present experiment). High pressure was applied with a compact hand pump (Syn Corporation TP-501).

2.3. TG Measurements. Details of the principle have been described elsewhere. ${ }^{27-30}$ Briefly, in the TG method, two laser pulses are introduced into the sample solution, which is incorporated into the high-pressure apparatus. The intensity (ITG) is proportional to the square of the generated refractive index change $(\delta n)$ arising from the temperature change (thermal grating, $\left.\delta n_{\text {th }}\right)$, volume change $\left(\delta n_{\mathrm{v}}\right)$, and absorption change $\left(\delta n_{\mathrm{pop}}\right)$. The experimental setup for the TG measurement was similar to that reported before. $^{24,26}$ The TG signals were monitored after photoexcitation by a $\mathrm{XeCl}$ excimer laser-pumped dye laser beam (Lambda Physik CompexPro102; $\lambda=308 \mathrm{~nm}$, Lumonics Hyper Dye 300; $\lambda=462 \mathrm{~nm}$ ). This light was detected by a photomultiplier tube (R1477, 
Hamamatsu, Japan). A CW diode laser (835 nm, Crysta Laser) was used as the probe beam. The grating wavenumber $q$ was determined from the decay rate of the thermal grating signal of a calorimetric reference sample (bromocresol purple in water) measured under the same conditions. The signal was fed into a digital oscilloscope (TDS-7104; Tektronix) and averaged, usually 20-100 times. The repetition rate for excitation was set to $0.04 \mathrm{~Hz}$, which is slower than the dark recovery time of TePixD $(\sim 5 \mathrm{~s}) .{ }^{25}$ Whenever the pressure was changed, we always reset the pressure to $0.1 \mathrm{MPa}$ to check the reproducibility of the signal. It was confirmed that the TG signals were always completely reproducible to the pressure change.

2.4. DLS Measurements. The DLS experiment was performed with a fiber-optics-based correlator (FDLS-3000, Otsuka Electronics). The sample solution was encapsulated in a high-pressure inner cell composed of quartz and silicone tubes (sample volume $\sim 300 \mu \mathrm{L}$ ), and that inner cell was placed inside the high-pressure apparatus. Continuous $532 \mathrm{~nm}$ laser light was transmitted through the tube, and the scattered light was detected by a photomultiplier tube at an angle of $90^{\circ}$. The autocorrelation function was averaged 300 times. The scattering vector was calculated by a similar method to that reported before. ${ }^{23}$

2.5. Densimetry under High Pressure. Density measurements were performed using a high-precision densimeter (DMA 512, Anton Paar, Graz, Austria), which was designed to measure the density under pressures up to $40 \mathrm{MPa}$. The period of harmonic oscillation of the cell reflecting the density was monitored by a universal counter (SC-7205, Iwatsu, Tokyo, Japan). The resolution of the period corresponded to a density of about $1 \times 10^{-6} \mathrm{~g} \mathrm{~mL}^{-1}$. Pressure was applied by pumping water with a hand pump (FHP-5, Riken Seiki, Tokyo, Japan) and a cylinder (S04-70, Riken Seiki). The pressure 
was monitored using a digital pressure gauge (DPS-700S, Riken Seiki) with an accuracy of $0.001 \mathrm{MPa}$. The temperature was controlled to within $0.001{ }^{\circ} \mathrm{C}$ using a thermocirculator (TC-100, Tokyo Riko, Tokyo, Japan), and was monitored with a digital thermometer (4600, YSI, USA). All data were fed to a personal computer at $10 \mathrm{~s}$ intervals via a RS-232C interface. The apparatus constant and density were determined according to previously described procedures. ${ }^{31}$

The apparent specific volume of the protein $\left(v^{*}\right)$ at a given pressure was calculated by the following equation:

$v^{*}=\left(1 / m^{*}\right)\left[1-\left(d_{\mathrm{s}}^{*}-m^{*}\right) / d_{0}^{*}\right]$

where $d_{0}{ }^{*}$ and $d_{\mathrm{s}}{ }^{*}$ are the densities of the solvent and solution at a given pressure, respectively, and $m^{*}$ is the protein concentration $\left(\mathrm{g} \mathrm{mL}^{-1}\right)$, which was calibrated to the pressure using the relationship $m^{*}=m\left(d_{\mathrm{s}}{ }^{*} / d_{\mathrm{s}}\right)$, where $m$ and $d_{\mathrm{s}}$ are the protein concentration and solution density, respectively, at atmospheric pressure. The $m$ value was determined within an accuracy of $0.1 \mathrm{mg} \mathrm{mL}^{-1}$ using the dry mass of the protein solution and solvent by thermogravimetry (TG/DTA 6200, Seiko Instruments, Japan). The obtained $m$ was approximately $13.7-14.8 \mathrm{mg} / \mathrm{mL}$ from the density measurements.

2.6. Cross-Linking Experiments under High Pressure. The oligomeric state of TePixD at high pressure was investigated by cross-linking experiments using glutaraldehyde (GA), which specifically reacts with a primary amine group (i.e., $\varepsilon$-amino groups of Lys residues) and forms stable covalent bonds among the inter- and intra-subunits. The TePixD solution $(330 \mu \mathrm{M})$ was mixed with GA and the final concentration of GA was set to $0.5 \mathrm{mM}$. The reaction mixture was incubated at 0.1 or $200 \mathrm{MPa}$ for $48 \mathrm{~h}$ at $23{ }^{\circ} \mathrm{C}$. For the cross-linking experiment at $200 \mathrm{MPa}$, the reaction mixture was encapsulated in the inner cell of the high-pressure apparatus used in the TG 
experiment. For both the experiments at 0.1 and $200 \mathrm{MPa}$, the experimental conditions were identical except for the pressure. The incubation was performed in the dark. The time from preparing the solutions to the start of the high-pressure experiment was less than $10 \mathrm{~min}$, which was sufficiently short for the cross-linking reaction. To stop the cross-linking reaction, $1 \mathrm{M}$ Tris- $\mathrm{HCl}$ buffer was added to the reaction mixture. The polypeptide samples were analyzed by sodium dodecyl sulfate polyacrylamide gel electrophoresis (SDS-PAGE). Polypeptide bands were visualized with Coomassie blue staining.

2.7. Calculation of the Protein Volume. The solvent excluded volume of TePixD was calculated from the X-ray crystal structure ${ }^{17}$ by NSOL program..$^{32}$ The distributions of the cavities within the TePixD decamer are calculated by the " $3 \mathrm{~V}$ : cavity, channel and cleft volume calculator and extractor" (3V) program on the web server, ${ }^{33}$ based on the same X-ray crystal structure. ${ }^{17}$ The probe radius used for the solvent water was $1.5 \AA$. In the calculations by $3 \mathrm{~V}$ program, a high grid resolution for the structure (voxel size 0.5 Å) was used.

\section{RESULTS}

3.1. Pressure-Dependent Reaction of TePixD. The reaction scheme of TePixD has been revealed by the TG method in a wide time range. ${ }^{22}$ The TG signal consists of three components: the thermal grating component (decay rate constant $D_{\text {th }} q^{2}$, thermal diffusivity $D_{\text {th }}$, and grating wavenumber $q$ ), a volume expansion process (weak decay after thermal grating signal with a time constant of $40 \mu \mathrm{s}$ ), and a peak of the molecular diffusion signal, which represents the change in the diffusion coefficient $(D)$. The reaction scheme of TePixD determined from the signal is shown in Figure 1. Figure 2 shows the pressure dependence of the molecular diffusion signals of TePixD at $q^{2}=7.0$ 
$\times 10^{10} \mathrm{~m}^{-2}$ at a weak light intensity of $1.02 \pm 0.02 \mathrm{~mJ} \mathrm{~cm}^{-2}$, which is weak enough to excite only one monomer unit in the decamer. ${ }^{34}$

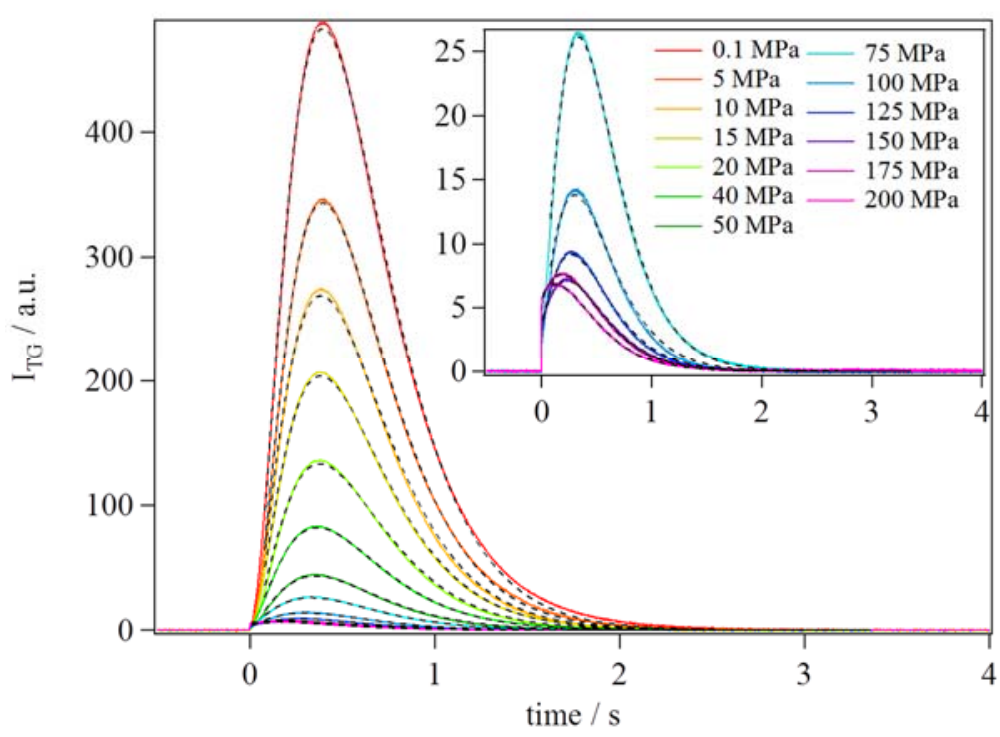

Figure 2. Molecular diffusion signal of TePixD measured at the grating wavenumber of $q^{2}=7.0 \times 10^{10} \mathrm{~m}^{-2}$ in the pressure range $0.1-200 \mathrm{MPa}$. The pressures are shown in the legend. The dashed lines show the best fitted curves using eq 4 . The inset shows the magnified signals in a high-pressure region.

Interestingly, the intensity of the signal drastically decreased with a slight increase in pressure. To investigate the origin of the pressure dependence of the signal without any ambiguity, we analyzed the signal as follows. First, we analyzed the signal at $0.1 \mathrm{MPa}$. Because the decamer and pentamer are in equilibrium in the solution, the diffusion signal should be expressed as a sum of the decamer and pentamer contributions:

$$
I_{T G}(t)=\alpha\left[\delta n_{10}(t)+\delta n_{5}(t)\right]^{2}
$$

where $\alpha$ is a constant, $\delta n_{10}(\mathrm{t})$ and $\delta n_{5}(\mathrm{t})$ are the time dependences of the refractive index 
changes after photoexcitation of the decamer and the pentamer, respectively. According to a previous study, ${ }^{22}$ the decamer contribution $\left(\delta n_{10}(t)\right)$ should be analyzed based on a model of

$$
\mathrm{R} \stackrel{\mathrm{h} \nu}{\rightarrow} \mathrm{I} \stackrel{k}{\rightarrow} \operatorname{Pr}
$$

where R, I, and Pr denote the reactant (decamer), intermediate, and product (pentamer), respectively, and $k$ is the rate constant of the dissociation reaction of the decamer. The molecular diffusion signal of the decamer $\left(\delta n_{10}(t)\right)$ based on this scheme is given by

$$
\begin{aligned}
& \delta n_{10}(t)=\delta n_{I} \exp \left\{-\left(D_{I} q^{2}+k\right) t\right\}+ \\
& \delta n_{P r} \frac{k}{\left(D_{P r}-D_{I}\right) q^{2}-k}\left[\exp \left\{-\left(D_{I} q^{2}+k\right) t\right\}-\exp \left(-D_{P r} q^{2} t\right)\right]-\delta n_{R} \exp \left(-D_{R} q^{2} t\right)
\end{aligned}
$$

where $D_{\mathrm{I}}, D_{\mathrm{Pr}}$, and $D_{\mathrm{R}}$ are the diffusion coefficients of the intermediate, product, and reactant, respectively, and $\delta n$ are the corresponding refractive index changes of these species.

At the grating wavenumber in Figure 2, because the protein diffusion signal appeared in a much slower time ( $\sim$ subsecond) than the dissociation reaction time of the decamer $\left(\sim 4 \mathrm{~ms}^{22}\right.$, as shown in section 3.2$)$, the effect of the reaction kinetics on the diffusion signal can be ignored (i.e., $k>>\operatorname{II}^{2}$ and $\exp (-k t) \approx 0$ in eq 2 ). Therefore, $\delta n_{10}(\mathrm{t})$ could be be simply expressed by the diffusion of the reactant and the product:

$$
\delta n_{10}(t)=\delta n_{P r} \exp \left(-D_{P r} q^{2} t\right)-\delta n_{R} \exp \left(-D_{R} q^{2} t\right)
$$

\section{(3)}

On the other hand, because photoexcitation of the pentamer does not exhibit any diffusion change, the signal owing to the pentamers $\left(\delta n_{5}(t)\right)$ is expressed by a single exponential function with a rate constant of $D_{5} q^{2}$, where $D_{5}$ is the diffusion coefficient of the pentamer. Because $D_{5}$ is the same as $D$ of the reaction product (pentamer) of the 
decamer $\left(D_{\mathrm{Pr}}\right)$ within our experimental accuracy (ref 23 and SI-1), the molecular diffusion signal of the pentamer is expressed by

$\delta n_{5}(t)=\delta n_{5} \exp \left(-D_{P r} q^{2} t\right)$

where $\delta n_{5}$ is the refractive index change of the pentamer reaction. Therefore, the molecular diffusion signal at pressure $P$ should be written as

$$
\begin{aligned}
I_{T G}(t)= & \alpha\left[\delta n_{10}(t)+\delta n_{5}(t)\right]^{2} \\
& =\alpha\left[\left(\delta n_{P r}(P)+\delta n_{5}(P)\right) \exp \left(-D_{P r} q^{2} t\right)-\right.
\end{aligned}
$$

$\left.\delta n_{R}(P) \exp \left(-D_{R} q^{2} t\right)\right]^{2}$

where we use $(P)$ on the right-hand side to clearly show the pressure-dependent amplitudes of these terms. To investigate the pressure effect on the reaction, at first, we determined $D_{\operatorname{Pr}}$ and $D_{\mathrm{R}}$ under the present conditions by biexponential fitting of the signal at $0.1 \mathrm{MPa}$ to eq 4 . To remove any ambiguity, we fixed the ratio of $D$ (i.e., $D_{\mathrm{R}} / D_{\mathrm{Pr}}$ ) to 1.5 , which was determined in a previous study. ${ }^{22}$ (If we did not pre-fix the ratio of $\mathrm{D}$, the fitting should be as good as Fig.2. This is a reason why we used the pre-fixed value for $D_{R} / D_{p r}$.) Using this restriction, $D_{\operatorname{Pr}}$ and $D_{R}$ at $0.1 \mathrm{MPa}$ were determined to be $2.9 \times 10^{-11} \mathrm{~m}^{2} \mathrm{~s}^{-1}$ and $4.5 \times 10^{-11} \mathrm{~m}^{2} \mathrm{~s}^{-1}$, respectively.

Next, the signals at various pressures were analyzed by fitting with the above-determined $D_{R}$ and $D_{\operatorname{Pr}}$ values, and the pressure-dependent amplitudes were determined. The pressure dependence of $D$ can be ignored because the viscosity within this pressure range is almost constant. ${ }^{35}$ The best fitted curves are shown as dashed lines in Figure 2, and the calculated signals generally reproduced the observed values very well. The determined pressure dependence of the fraction of the decamer that exhibits the diffusion signal (reactive decamer), $f(P)=\delta n_{\mathrm{R}}(P) / \delta n_{\mathrm{R}}(0.1)$, where $\delta n_{\mathrm{R}}(0.1)$ is the value at $0.1 \mathrm{MPa}$, is plotted against pressure in Figure 3. It is apparent that the 
amplitude that represents the reactant contribution $\left(\delta n_{R}\right)$ to the diffusion change significantly decreased with increasing pressure even in a relatively low pressure range.

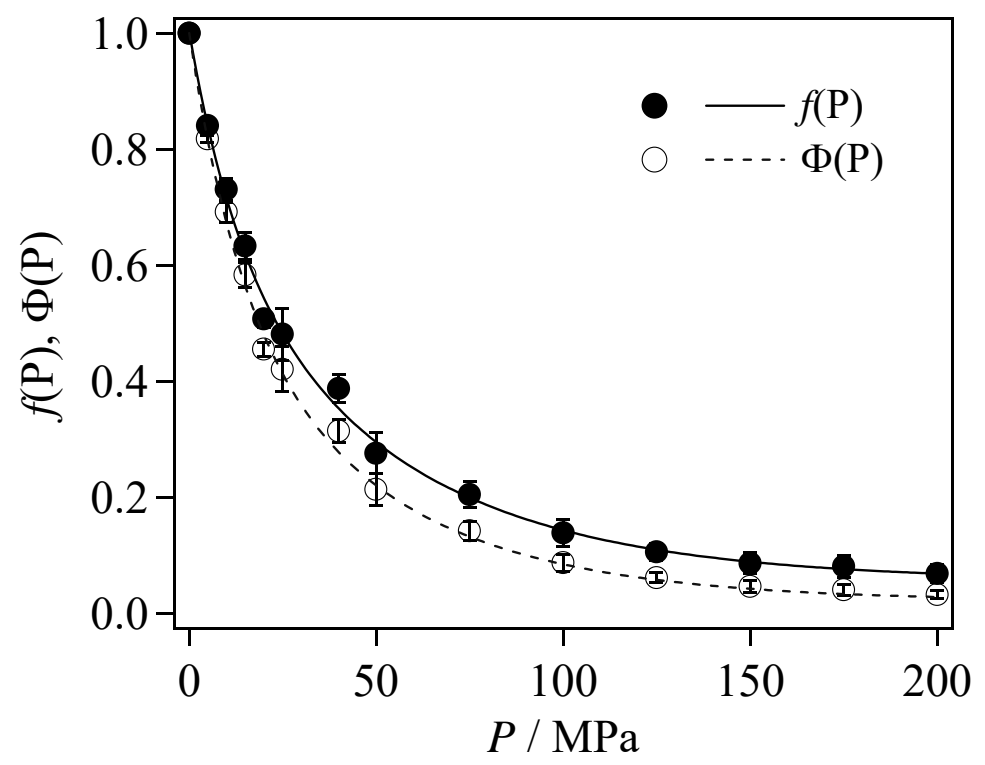

Figure 3. Pressure dependence of the relative amplitude of the reactant $(f(P), \bullet)$ determined from the TG signal in Figure 2. Open circles $(\circ)$ are the pressure-dependent relative quantum yield of the dissociation reaction $(\Phi(P))$ normalized by $\Phi(0.1)$. The solid and dashed lines are guides for eye for $f(P)$ and $\Phi(P)$, respectively.

3.2. Pressure-Dependent Kinetics of the Reaction. From the signal at the low $q^{2}$ in section 3.1, we found that the amplitude of the diffusion signal significantly decreased by applying external pressure. This suggests that the amount of reaction decreased with pressure. However, to confirm this, the pressure dependence of the reaction rate needs to be investigated because the signal intensity might decrease because of a decrease of the reaction rate. To investigate the effect of pressure on the kinetics, we measured the 
diffusion signal at a larger grating wavenumber of $q^{2}=5.8 \times 10^{12} \mathrm{~m}^{-2}$ at various pressures. At this grating wavenumber, the diffusion signal appeared in several milliseconds, which is close to that of the dissociation reaction rate $(\sim 4 \mathrm{~ms}$ at 0.1 $\mathrm{MPa}){ }^{22}$ so the temporal profile reflects the reaction kinetics. Similar to the signal measured in the slower time range shown in the section 3.1, the signal intensity decreased with increasing pressure (Figure 4).

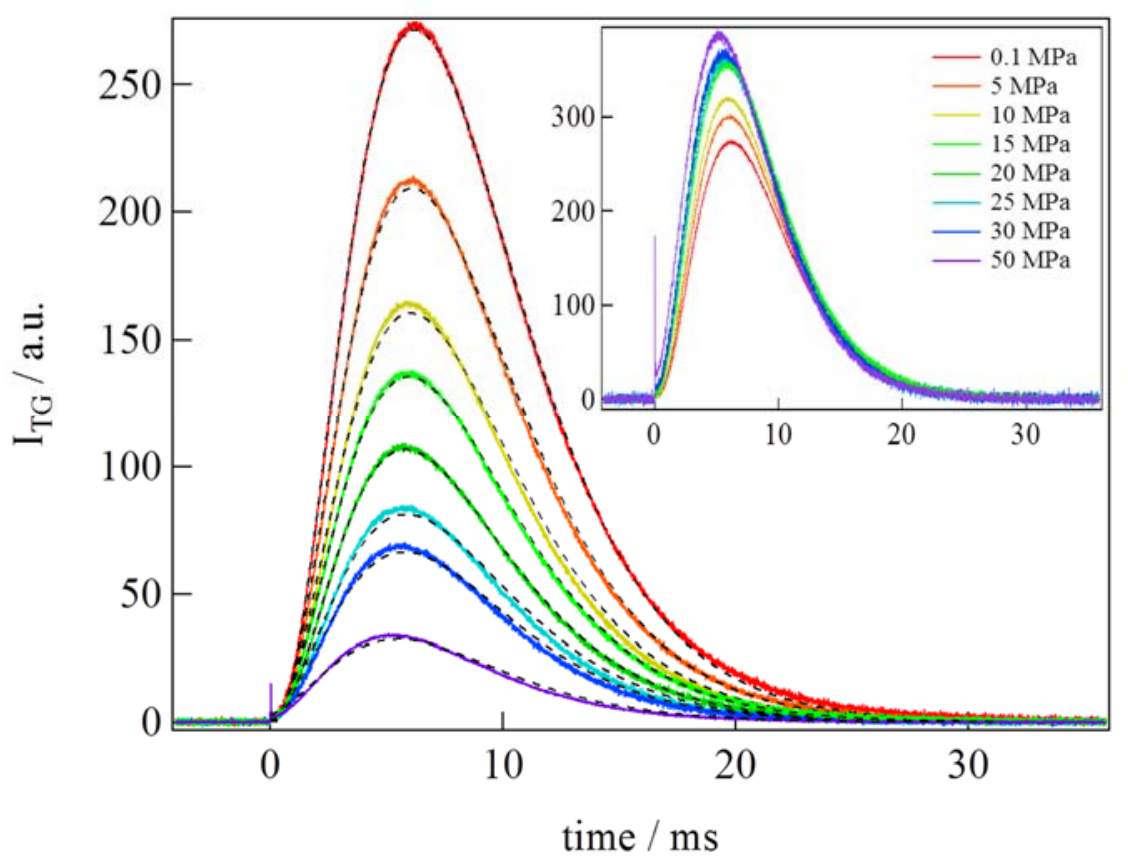

Figure 4. Molecular diffusion signal of TePixD measured at the grating wavenumber of $q^{2}=5.8 \times 10^{12} \mathrm{~m}^{-2}$ for $P \leq 50 \mathrm{MPa}$. The dashed lines show curves fitted using eq 5 . The inset shows the signals normalized by $f(P)$ in Figure 3.

However, when the signals were normalized by the signal intensity measured at the low $q^{2}(f(P)$ in Figure 3), we found that the signal intensity increased with increasing pressure (Figure 4, inset). This tendency can be explained by the pressure dependence of the reaction rate. Because the signal intensity should be weak before the reaction 
begins and gradually increase as the reaction proceeds, this increase must be because of increase of the reaction rate. To extract kinetic information, we analyzed the signal using

$$
I_{T G}(t)=\alpha\left[f(P) \delta n_{10}(0.1)(t)+\delta n_{5}(P) \exp \left(-D_{P r} q^{2} t\right)\right]^{2}
$$

where $\delta n_{10}(0.1)(t)$ is a function of eq 2 at $0.1 \mathrm{MPa}$, and the pressure-dependent amplitude was taken into account by the factor $f(P)$. First, we fitted the signal at 0.1 MPa using previously reported values of $k=250 \mathrm{~s}^{-1}$, ratios of the diffusion coefficients $D_{\mathrm{R}} / D_{\operatorname{Pr}}=1.5$ and $D_{\mathrm{I}} / D_{\mathrm{R}}=0.9,{ }^{22} D_{\mathrm{R}}=4.5 \times 10^{-11} \mathrm{~m}^{2} \mathrm{~s}^{-1}$ (determined in section 3.1), and $f(0.1)=1$. Using these values, we determined the relative refractive index changes $\left(\delta n_{\mathrm{R}}\right.$, $\delta n_{\mathrm{I}}$, and $\delta n_{\mathrm{Pr}}$ ) in $\delta n_{10}(0.1)(t)$ (eq 2). To fit signals at higher pressures using eq $5, D, \delta n_{\mathrm{R}}$, $\delta n_{1}$, and $\delta n_{\mathrm{Pr}}$ were fixed to the determined values at $0.1 \mathrm{MPa}$, and $f(P)$ was also fixed to the predetermined values in Figure 3. Therefore, fitting to eq 5 now has only two adjustable parameters, $k$ and $\delta n_{5}(P)$, for the analysis at higher pressures. Because of this constraint, $k$ was uniquely determined at each pressure. The pressure dependence of $k$ determined in the pressure range $0.1-50 \mathrm{MPa}$ is shown in Figure 5 (it was difficult to uniquely determine $k$ above $50 \mathrm{MPa}$ because the molecular diffusion signal was too weak for accurate determination). 


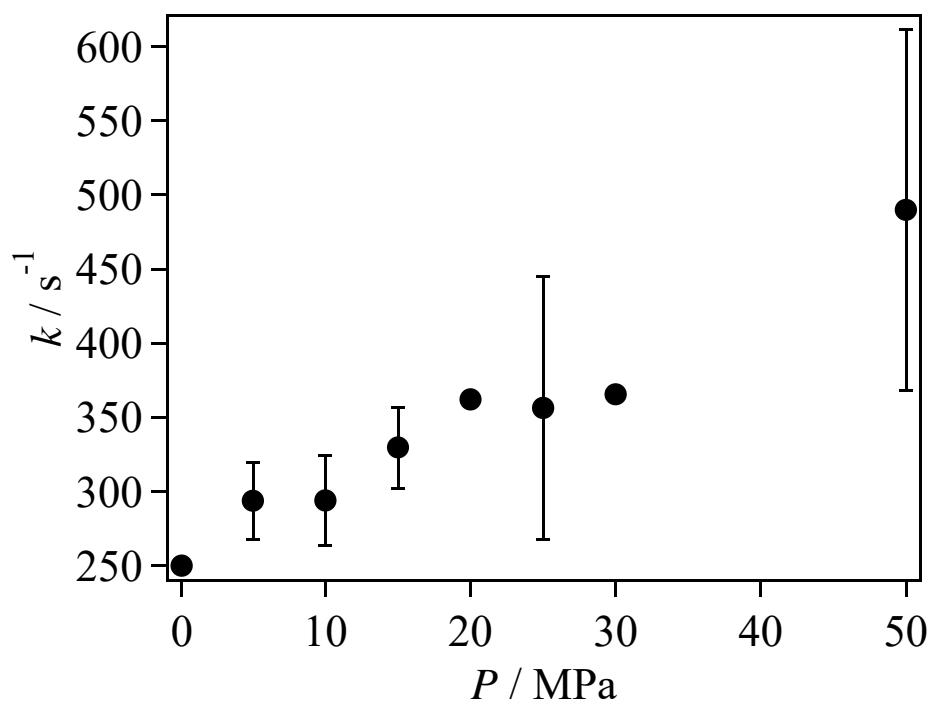

Figure 5. Pressure dependence of the dissociation reaction rate $(k)$ in the pressure range $\leq 50 \mathrm{MPa}$.

It is interesting that the reaction rate increased with increasing pressure. Although the amplitude of the signal significantly decreased, the reaction rate $k$ showed relatively weak pressure dependence ( $k$ increased by less than two times for $P \leq 50 \mathrm{MPa}$ ). From the pressure dependence of the reaction rate $k$, the activation volume $\left(\Delta V^{\ddagger}\right)$ of this dissociation reaction was calculated to be $-30 \mathrm{~cm}^{3}$ (mol of decamer) ${ }^{-1}$.

\subsection{Origin of the Pressure Dependence: Pressure-Dependent Equilibrium Shift.}

As shown in section 3.1 and 3.2, the diffusion signal intensity drastically decreased by applying pressure even in the relatively low pressure region. The kinetics measurements indicate that this is not because of kinetics. There may be two possible origins of the pressure effect. First, because only the decamer undergoes the diffusion change reaction, the signal intensity may decrease because of a decrease in the population of the decamer. The decamer and pentamer are in equilibrium. Hence, if the equilibrium shifts to the 
pentamer by applying pressure, the decrease of the intensity of the diffusion signal can be reasonably explained. Second, the decrease may indicate a decrease of the reaction quantum yield of the $D$ change reaction (dissociation reaction) by pressure. We examine the possibility of the equilibrium shift below.

If the disappearance of the molecular diffusion signal of TePixD at $200 \mathrm{MPa}$ (Figure 2) is due to equilibrium shift from the decamer to the pentamer, almost all of all decamer should be dissociated into the pentamer at $200 \mathrm{MPa}$, and the decamer should not exist in the dark state at this pressure. We investigated the presence of the decamer by cross-linking experiments. The TePixD sample solutions were treated by the cross-linking reagent glutaraldehyde at 0.1 and $200 \mathrm{MPa}$, and analyzed by SDS-PAGE. The results are shown in the Supporting Information (SI-2). Cross-linked products of oligomers larger than the pentamer were detected at both 0.1 and $200 \mathrm{MPa}$, showing that the decamer still exists even under high pressure. This observation indicates that the disappearance of the molecular diffusion signal at $200 \mathrm{MPa}$ is not because of equilibrium shift to the pentamer.

For more quantitative analyses, we used the DLS technique at high pressures. Because the $D$ values of the pentamer and the decamer are clearly different $(D=4.5 \times$ $10^{-11} \mathrm{~m}^{2} \mathrm{~s}^{-1}$ (decamer) and $2.9 \times 10^{-11} \mathrm{~m}^{2} \mathrm{~s}^{-1}$ (pentamer)), the population at the dark state can be measured by the diffusion detection. Figure 6 shows autocorrelation profiles of the DLS signals measured at 0.1 and $150 \mathrm{MPa}$ at a scattering wavenumber of $q^{2}=4.97 \times 10^{14} \mathrm{~m}^{-2}$. 


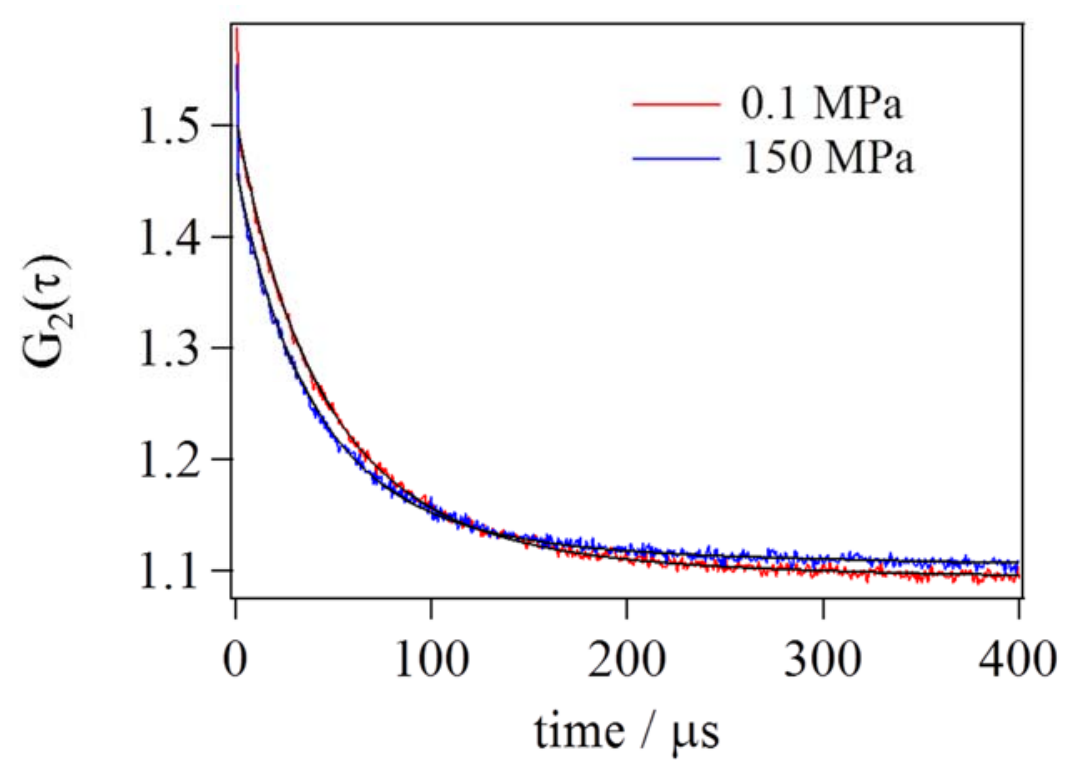

Figure 6. Autocorrelation profiles of light scattering of the TePixD solution measured at 0.1 (red) and $150 \mathrm{MPa}$ (blue) at a scattering wavenumber of $q^{2}=4.97 \times 10^{14} \mathrm{~m}^{-2}$. Black solid lines are the best fitted curves using eq 6 .

Because this autocorrelation profile should be expressed by the diffusion of the decamer and the pentamer, these profiles were fitted using the following function:

$$
G_{2}(\tau)=1+\left[a \exp \left(-D_{R} q^{2} \tau\right)+b \exp \left(-D_{5} q^{2} \tau\right)+c \exp \left(-k_{3} \tau\right)\right]^{2}
$$

where $a, b$, and $c$ are pre-exponential factors. The last term of the right-hand side represents the contribution of unavoidable dust in the solution. The rate constant $k_{3}$ is much ( $\sim 100$ times) smaller than the other rate constants, and hence this term can be easily separated from the other terms. In this expression, $a$ and $b$ represent the relative amplitudes of the decamer and pentamer contributions, respectively. As stated above, $D 5$ should be the same as $D_{\operatorname{Pr}}$, so $D_{\operatorname{Pr}}$ and $D_{\mathrm{R}}$ were fixed to the values determined by the TG diffusion signal $\left(D_{\mathrm{R}}=4.5 \times 10^{-11} \mathrm{~m}^{2} \mathrm{~s}^{-1}\right.$ and $\left.D_{\operatorname{Pr}}=2.9 \times 10^{-11} \mathrm{~m}^{2} \mathrm{~s}^{-1}\right)$ in section 3.2. The adjustable parameters are the amplitudes $(a, b$, and $c)$ and $k_{3}$. The typical best fitted 
curves are shown in Figure 6. Qualitatively, because the autocorrelation profile at 150 MPa decays faster than that at $0.1 \mathrm{MPa}$, the population of the faster diffusive species (decamer) is larger at $150 \mathrm{MPa}$.

Quantitatively, using $a$ and $b$, the fraction of the decamer $\left(f_{10}\right)$ is expressed as

$$
f_{10}=a /(a+b)
$$

The values at various pressures are plotted in Figure 7 . Figure 7 shows that $f_{10}$ increased with increasing pressure, which indicates that the dark-state equilibrium between the decamer and pentamer shifted toward the decamer with increasing pressure.

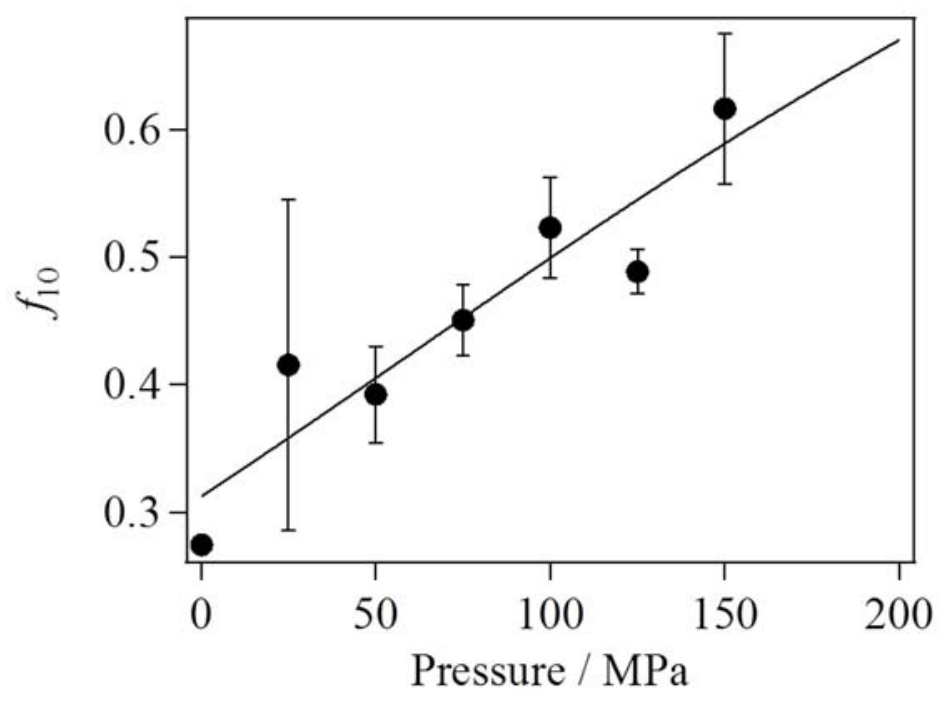

Figure 7. Pressure dependence of the fraction of the decamer $\left(f_{10}\right)$ determined from the DLS signals. The solid line is the fitting curve using eq 8 .

The pressure dependence of $f_{10}$ was also analyzed. Using the degree of dissociation of the decamer $(\alpha), f_{10}$ can be written as (also see SI-3)

$$
f_{10}=\frac{1-\alpha}{1+\alpha}
$$

This $\alpha$-value at a pressure $P$ is given by 
$\alpha=\frac{-m+\sqrt{m^{2}+16 m}}{8}$

$m=\frac{K_{d i s}}{C} \exp \left(-\frac{(P-0.1) \Delta V_{d i s}}{R T}\right)$

where $C$ is the total decamer concentration, $K_{\text {dis }}$ is the dissociation constant from the decamer to the pentamer at $0.1 \mathrm{MPa}$, and $\Delta V_{\text {dis }}$ is the reaction volume (see SI-3 for its derivation). We fitted the pressure dependence of $f_{10}$ (Figure 7) using the two adjustable parameters $K_{\text {dis }} / C$ and $\Delta V_{\text {dis. }}$ The fitting curve is shown as the continuous black line in the figure, and the parameters were uniquely determined as $K_{\mathrm{dis}} / C=2.31$ and $\Delta V_{\mathrm{dis}}=30$ $\pm 5 \mathrm{~cm}^{3}$ (mol of decamer) $)^{-1}$. This result reflects the molecular volume expansion upon dissociation, that is, the partial molar volume of the product (two pentamers) is larger than that of the reactant (decamer).

3.4. Volume Change upon Photodissociation Reaction. In principle, the volume change upon dissociation should be detected from the TG measurement, because the volume change affects the TG signal intensity. However, the signal intensity due to the expected volume change is very small, and the strong diffusion signal masks the grating signal coming from this volume changes. Here, to detect the volume grating component, we measured the TG signal at $q^{2}=4.5 \times 10^{10} \mathrm{~m}^{-2}$ (Figure 8). 


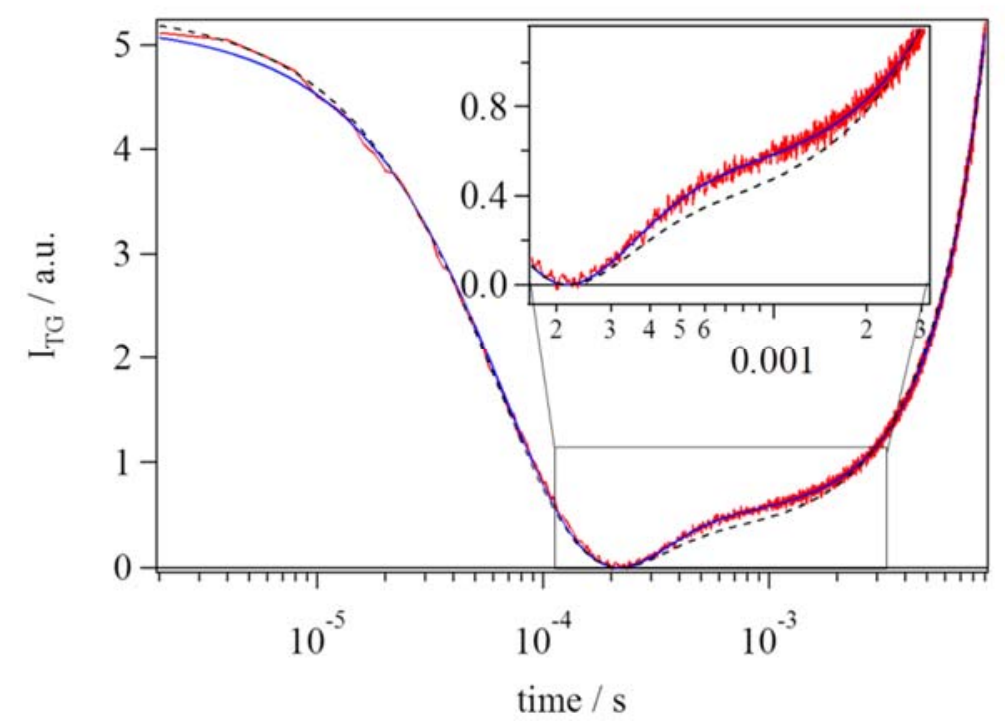

Figure 8. TG signal of TePixD at the grating wavenumber $q^{2}=4.5 \times 10^{10} \mathrm{~m}^{-2}$ at 0.1 MPa. The inset shows the magnified TG signal to highlight the volume contribution of the dissociation reaction. The solid and dashed lines are the best fitted curves using eq 9 with and without the volume change contribution, respectively.

At this specific grating wavenumber, the dissociation reaction component ( $\sim 4 \mathrm{~ms})$ is relatively separate from the thermal grating signal (decay in several $10 \mu \mathrm{s}$ ) and molecular diffusion signal components (slow increase observed in the millisecond time region). This TG signal is composed of three components: the thermal signal, the volume expansion signal with a time constant of $40 \mu \mathrm{s}$, and molecular diffusion. To extract the amplitude of the volume grating signal from the signal, the TG signal needs to be accurately analyzed. For this purpose, we reduce the ambiguity of the fitting using the equation

$$
\begin{aligned}
I_{T G}(t)=\alpha\left[\delta n_{t h} \exp \left(-D_{t h} q^{2} t\right)+\delta n_{V}\right. & \exp \left(-k_{V} t\right) \\
& +f(P)\left(\delta n_{I}-\delta n_{P r}\right) \exp (-k t)+
\end{aligned}
$$

$A+B t]^{2}$ 
where the first term represents the thermal grating, the second term represents the volume expansion, and the last two terms represent the contribution of the molecular diffusion signal in this short time range (see SI-4 for derivation). Because the refractive index depends on the volume (see SI-5) and the absorption spectrum does not change after red-shifted species formation, the refractive index change by dissociation $\left(\delta n_{\mathrm{I}}-\delta n_{\mathrm{Pr}}\right)$ represents the volume change upon the dissociation reaction.

We accurately determined the magnitude of $\delta n_{1}-\delta n_{\operatorname{Pr}}$ from the signal in the following way. First, the decay rate constant of the thermal grating $D_{\mathrm{th}} q^{2}$ was fixed to the value determined by the calorimetric reference signal. Second, to reduce ambiguity in the longer time region, $k_{V}$ and $k$ were fixed to the literature values $\left(40 \mu \mathrm{s}^{-1}\right.$ and 4 $\mathrm{ms}^{-1}$, respectively $)^{22}$. Furthermore, $\delta n \mathrm{v}$ was fixed to the value calculated from the reported volume change $\left(4 \mathrm{~cm}^{3} \mathrm{~mol}^{-1}\right) \cdot{ }^{24}$ If we assume that there is no volume change upon dissociation, that is, $\delta n_{\mathrm{I}}-\delta n_{\mathrm{Pr}}=0$, the best-fitted signal cannot reproduce the signal, as shown by the dashed line in Figure 8. Therefore, we must consider the volume change upon this reaction. The best fitted curve using eq 9 is shown by the solid line in Figure 8 . We obtained $\delta n_{\mathrm{I}}-\delta n_{\mathrm{Pr}}>0$, indicating that the volume change from the decamer to the (two) pentamers was positive. Its magnitude was calculated to be $\Delta V_{\text {dis }}{ }^{*}=19 \pm 1$ $\mathrm{cm}^{3}$ (mol of decamer) $)^{-1}$ by the method described in the Supporting Information (SI-5). The slight difference between the volume change from the DLS $\left(\Delta V_{\text {dis }}\right)$ and TG $\left(\Delta V_{\text {dis }}{ }^{*}\right)$ experiments is not surprising, because the volume change from the DLS experiment represents the volume difference between the decamer and the two pentamers in the ground state, while the value from the TG experiment represents the volume difference between the decamer and one pentamer in the excited state and one in the ground state.

We then determined $\Delta V_{\mathrm{dis}}{ }^{*}$ at various pressures. The pressure dependence of $\Delta V_{\mathrm{dis}}{ }^{*}$ is 
shown in Figure 9. From the slope, the isothermal compressibility change $\left(\Delta \kappa_{T}=\right.$ $\left.-(\partial \Delta V / \partial P)_{T}\right)$ upon dissociation was determined to be $\Delta \kappa_{\Gamma}=(2.1 \pm 0.1) \times 10^{-1} \mathrm{~cm}^{3}(\mathrm{~mol}$ of decamer $)^{-1} \mathrm{MPa}^{-1}$. The positive compressibility indicates that the fluctuation of the (two) pentamers is larger than that of the decamer.

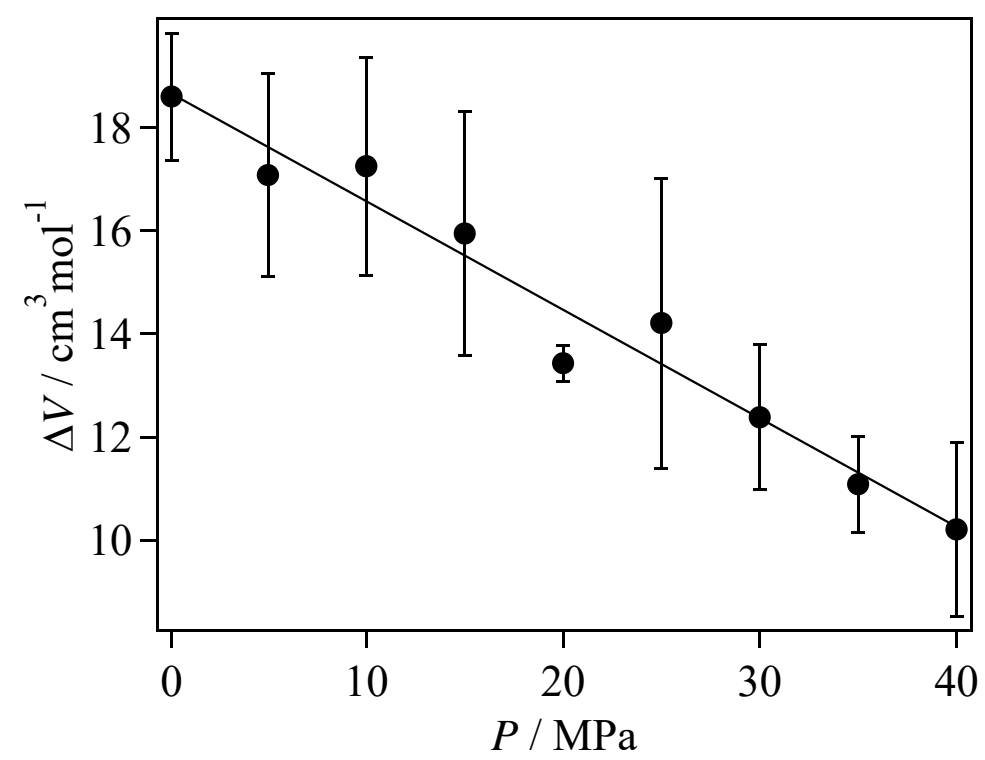

Figure 9. Pressure dependence of the reaction volume change of the dissociation reaction obtained from the TG signals. The solid straight line is the best-fitted line with a linear function of $P$. The slope of this line provides the compressibility change.

3.5. Pressure-Dependent Reaction Yield. In section 3.3, we found that the population of the decamer increased with increasing pressure. Hence, the decrease of the diffusion signal with pressure cannot be because of the decrease of the reactant (decamer), and it must be due to the quantum yield change of the dissociation reaction of the decamer. The pressure dependence of the reaction quantum yield $(\Phi)$ was calculated from $f(P)$ (Figure 3) by taking into account the increase of the decamer 
population with increasing pressure $\left(f_{10}\right)$ given by eq 8 . The values relative to $0.1 \mathrm{MPa}$ are shown in Figure 3 by open circles.

According to the reaction scheme of TePixD (Figure 1), the photoexcited TePixD initially gives the spectrally red-shifted intermediate species, and then a conformation change (volume change) and dissociation reaction occurs. Previously, it was reported that the pressure dependence of the yield of the red-shifted species is small, and the volume change reaction with $40 \mu$ s time constant does not depend on the oligomeric state regardless of whether TePixD is in the decamer form or pentamer forms. ${ }^{22,24}$ Therefore, the observed significant pressure dependence of the dissociation reaction occurs after the $40 \mu$ s conformation change.

One possible origin of the pressure-dependent quantum yield for the dissociation reaction could be a pressure induced conformation change of the monomer unit. However, because the absorption spectrum does not change at all with applied pressure until $200 \mathrm{MPa},{ }^{24}$ the conformation change at least around the chromophore can be ignored. In many protein systems, secondary structure changes are observed for $P>500$ $\mathrm{MPa} .{ }^{36}$ The tertiary structure often starts to change at $P>200 \mathrm{MPa} \cdot{ }^{37}$ Hence, in the relatively low pressure range below $200 \mathrm{MPa}$, we consider that volume changes originating from the cavities of the protein are important, because cavities can be easily compressed. ${ }^{38-43}$ If this is the case, we may see pressure-dependent compressibility $\left(\kappa_{\mathrm{T}}\right)$ because the presence of cavities enhances $\kappa_{\mathrm{T}}$.

The above discussion may be reasonable because of the following consideration. Previously, we reported that the dissociation reaction is controlled by fluctuation. ${ }^{24}$ This conclusion was drawn because of an observed correlation between the light-intensity-dependent reaction yield and the fluctuation. The TePixD decamer cannot 
undergo dissociation when its multiple monomers are simultaneously excited under strong excitation. ${ }^{34}$ We also showed that the compressibility change of the TePixD decamer becomes smaller under multiple excitations (i.e., when the dissociation reaction is suppressed) than the case of one monomer excitation (i.e., when the reaction occurs). Considering the importance of the fluctuation, we suspect that the pressure-dependent compressibility may be a cause of the observed pressure-dependent reactivity. In this case, the absolute value of the compressibility, not the relative value from the ground state, could be important. To test this possibility, we measured the protein volume at various pressures by the densimetric approach.

Figure 10 shows the specific volume of TePixD at various pressures. The slope of the volume versus pressure plot corresponds to the compressibility кт. Although it is very difficult to measure the protein volume, particularly as a function of pressure, and the data points are rather scattered, the pressure dependence of the volume (Figure 10) is not linear, indicating pressure-dependent compressibility.

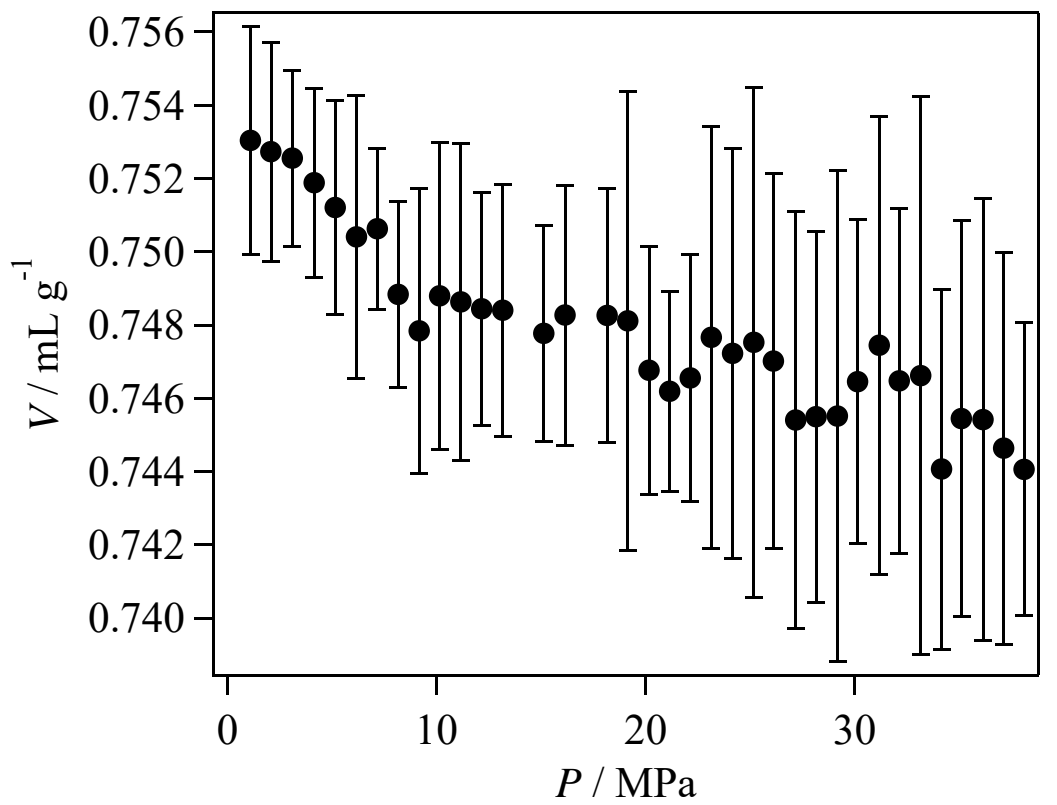


Figure 10. Specific volume of TePixD measured at various pressures.

Because the slope decreased with increasing pressure, it is reasonable to assume that the compressibility of TePixD decreases with increasing pressure. For example, the compressibility calculated from the data in the range $0.1-10 \mathrm{MPa}$ was $98 \mathrm{~cm}^{3}$ (mol of decamer $)^{-1} \mathrm{MPa}^{-1}$, whereas that in a range 20-30 $\mathrm{MPa}$ was $19 \mathrm{~cm}^{3}(\mathrm{~mol} \text { of decamer })^{-1}$ $\mathrm{MPa}^{-1}$. This pressure-dependent compressibility should contain both decamer and pentamer contributions. However, because the compressibility change from the decamer to the pentamers determined in section $3.4\left(\Delta \kappa_{\Gamma}=0.21 \mathrm{~cm}^{3} \mathrm{~mol}^{-1} \mathrm{MPa}^{-1}\right)$ is small, and the fraction of the decamer $\left(f_{10}\right)$ only slightly increased at $30 \mathrm{MPa}$ (see Figure 7 ), we consider that this pressure dependence reflects the compressibility decrease of the decamer by pressure. Because the compressibility is directly related to the root mean square of the volume fluctuation by

$\left(\delta V_{r m s}\right)^{2}=R T \kappa_{T}$

where $R$ is the gas constant, $T$ is the temperature, and $\delta V_{r m s}=\sqrt{\left\langle(V-\langle V\rangle)^{2}\right\rangle},(\langle V\rangle$ is the average of quantity $V),{ }^{44}$ the smaller $\kappa_{\mathrm{T}}$ at higher pressures means that the structural fluctuation of the TePixD decamer (and pentamer) was suppressed by the pressure.

\section{DISCUSSION}

We found that the equilibrium of the TePixD oligomers shifts to the decamer by applying pressure, indicating a positive volume change upon dissociation. Indeed, this volume change was determined to be $\Delta V_{\mathrm{dis}}=30 \mathrm{~cm}^{3} \mathrm{~mol}^{-1}$. Interestingly, however, in many cases, protein oligomers are likely to dissociate into subunits under high pressure, because of the negative volume change upon dissociation, which is often explained by 
the disappearance of cavities at the interface of the subunits and the hydration change. ${ }^{37,45}$ On the other hand, if the protein is a rigid sphere, the positive volume change upon dissociation is natural, because the solvent exclusion volume at the interface increases upon dissociation. We calculated the volume difference between two pentamers and one decamer based on the crystal structure of the TePixD decamer using the NSOL program. ${ }^{32}$ Using the crystal structure $(1 \mathrm{X} 0 \mathrm{P} \text { in PDB })^{17}$, the solvent excluded volumes $^{46,47}$ ( $V_{\text {ex's, }}$, defined in Figure 11) of two pentamers were calculated to be 80567 and $81330 \mathrm{~cm}^{3} \mathrm{~mol}^{-1}$ (the reported structures of the two pentamer rings are slightly different).

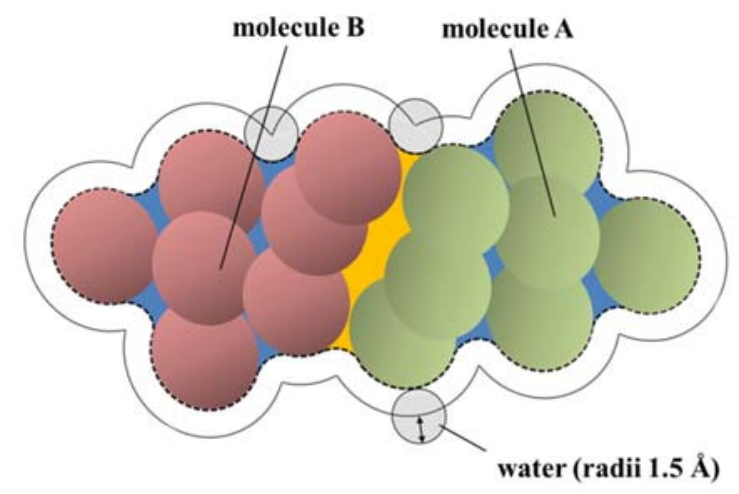

— : solvent accessible surface

..... : molecular surface

$\square$ : inside the 'solvent accessible surface' is

defined as 'solvent excluded volume $\left(\mathrm{V}_{\mathrm{ex}}\right)$ '

: Van der Waals volume of molecule A

: Van der Waals volume of molecule B

: cavities inside the molecule $A$ or $B$

: cavities between molecules $A$ and $B$

Figure 11. Schematic illustration of the molecular and solvent accessible surfaces, intra and inter-molecular cavities, and van der Waals and solvent exclusion volumes of the two proteins A and B with a spherical water probe (radius $1.5 \AA$ ).

Taking the sum of these two values, $V_{\text {ex }}$ of the two pentamer rings is $161897 \mathrm{~cm}^{3}$ $\mathrm{mol}^{-1}$. $V_{\mathrm{ex}}$ of the decamer calculated using the same program is $158184 \mathrm{~cm}^{3} \mathrm{~mol}^{-1}$. Therefore, the volume change by dissociation using the crystal structure is $\Delta V_{\text {dis }}=3713$ 
$\mathrm{cm}^{3} \mathrm{~mol}^{-1}$, which is very large compared with the observed volume change. This difference may originate from possible two contributions; one is the disappearance of the cavities that may exist between the two pentamer rings, and the other is the hydration effect at the interface between the two pentamer rings. Of course, by the disappearance of the cavities, the volume change upon the dissociation should decrease. Furthermore, since the partial molar volume of the hydrated water molecule is smaller than that of the bulk water molecule, ${ }^{48-50}$ the hydration effect also contributes to decrease the volume change. Indeed, the strong hydration was suggested previously from the observation of the small diffusion coefficient of the TePixD pentame $(D=3.2$ $\left.\times 10^{-11} \mathrm{~m}^{2} \mathrm{~s}^{-1}\right)$ compared with that of the decamer $\left(D=4.9 \times 10^{-11} \mathrm{~m}^{2} \mathrm{~s}^{-1}\right)$. These two contributions may be a cause of the observed small volume change upon the dissociation.

However, these two changes cannot be sole changes upon the dissociation. The disappearance of the cavities and increase of the hydration generally cause a decrease of the compressibility. ${ }^{38,51}$ Hence, both of these contribution cannot explain the observed compressibility increase; $\Delta \kappa_{\mathrm{T}}=0.21 \mathrm{~cm}^{3} \mathrm{~mol}^{-1} \mathrm{MPa}$. In order to explain the observed positive change in the compressibility, we must consider that the compressibility (or the fluctuation) of the pentamer ring increases upon the dissociation. Probably, the cavities within the pentamer ring increases upon the dissociation. A volume change upon change of the intermolecular interactions is not unusual. It has been reported that the binding of a ligand to a protein dramatically changes its internal packing and cavity volume. ${ }^{39,52}$ For example, in the case of Escherichia coli dihydrofolate reductase, its cavity volume decreases from 151 to $90 \mathrm{~cm}^{3} \mathrm{~mol}^{-1}$ upon binding of folate. If the cavity of the pentamer increases upon dissociation, the internal packing of the pentamer would be looser upon 
dissociation from the other pentamer unit. The increase of the cavity volume upon dissociation is consistent with the increase of $\kappa_{\mathrm{T}}$ upon dissociation $\left(\Delta \kappa_{\mathrm{T}}=0.21 \mathrm{~cm}^{3}\right.$ $\mathrm{mol}^{-1} \mathrm{MPa}$ ). In contrast to the positive reaction volume, the activation volume of the dissociation reaction was negative $\left(\Delta V^{\ddagger}=-30 \mathrm{~cm}^{3} \mathrm{~mol}^{-1}\right)$, which might mean that the conformation change of the pentamer unit is not complete in the transition state.

Compressibility is a thermodynamic parameter that is directly related to volume fluctuation (eq 10), although this relationship is only strictly applicable to the intrinsic volume and not the specific volume. ${ }^{53}$ However, we discuss the magnitude of the volume fluctuation using this relationship because it has been used to discuss the fluctuation of proteins and is frequently found to correlate with the reactivity. ${ }^{38,51,52,54,55}$ In the pressure range from 0.1 to $10 \mathrm{MPa}$, the compressibility was $98 \mathrm{~cm}^{3} \mathrm{~mol}^{-1} \mathrm{MPa}^{-1}$, and $\delta V_{\text {rms }}$ calculated from this $\kappa_{\mathrm{T}}$ is $490 \mathrm{~cm}^{3} \mathrm{~mol}^{-1}$. This volume fluctuation $\left(490 \mathrm{~cm}^{3}\right.$ $\mathrm{mol}^{-1}$ ) is $0.38 \%$ of the partial molar volume of the TePixD decamer. Similarly, the volume fluctuation is $0.17 \%$ of the partial molar volume in the pressure range $20-30$ $\mathrm{MPa}$. It was reported that the $\delta V_{\text {rms }}$ values of 24 globular proteins were widely dispersed in the range $0.12 \%-0.54 \%$ of the total protein volumes, ${ }^{37}$ and was about $0.3 \%$ on average. ${ }^{38,51}$ The magnitude of the volume fluctuations obtained here $(0.38 \%$ at $0.1 \mathrm{MPa}$ and $0.17 \%$ at $30 \mathrm{MPa}$ ) are within this range of variation. Interestingly, this means that the structure of TePixD changes from a relatively "soft" globular protein to a relatively "hard" globular protein.

It is difficult to believe that the observed large pressure-dependent compressibility within $30 \mathrm{MPa}$ originated from a large (secondary or ternary) structure change of the monomer unit in this small pressure range. ${ }^{36,37,41}$ Because protein-protein interactions are generally more sensitive to pressure than the tertiary structure, ${ }^{37}$ we believe that this 
significant compressibility decrease originates from the pressure effect on the cavities at the interfaces of the monomer units. The TePixD decamer possesses two types of interfaces between the monomer units: the interface between two pentamer rings (H-interface) and the interface between the monomer units within the pentamer ring (V-interface) (Figure 12).

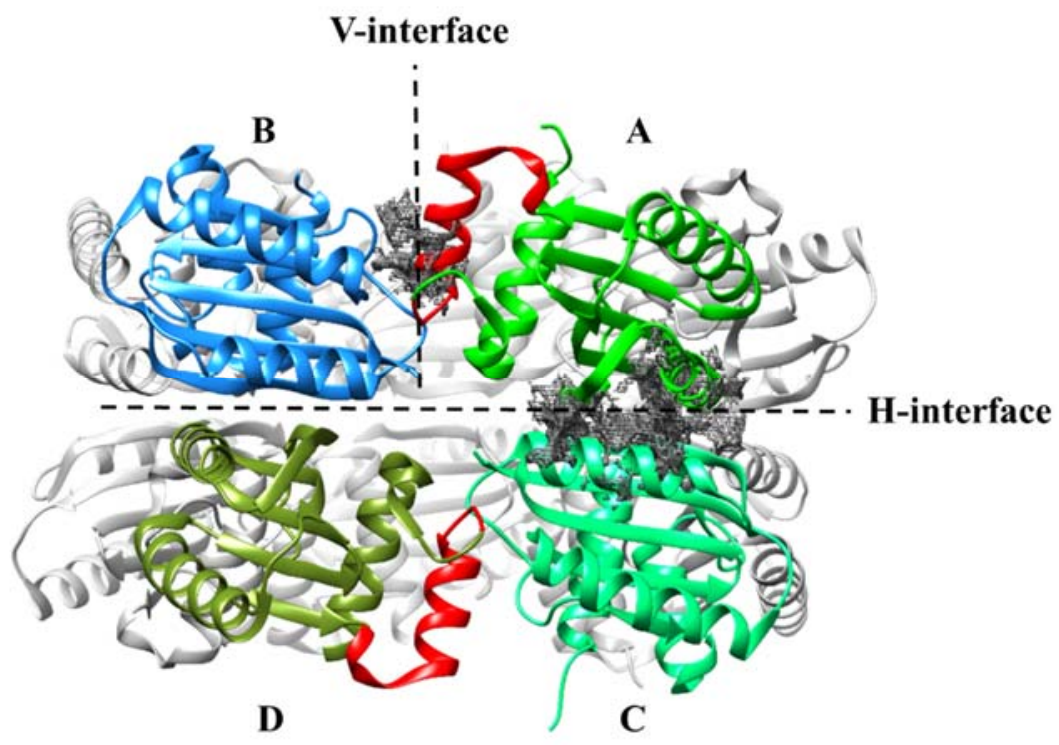

Figure 12. Side view of the TePixD decamer. Cavities between the monomer units from the $3 \mathrm{~V}$ program are shown by the gray region. For clarity, the cavities between monomers $\mathrm{A}$ and $\mathrm{B}$ (V-interface), and monomers $\mathrm{A}$ and $\mathrm{C}$ (H-interface) are shown. The C-terminal $\alpha 3$-helices of monomers A and D are shown in red.

The distributions of the cavities at such interfaces are calculated by the $3 \mathrm{~V}$ $\operatorname{program}^{33}$ and shown in Figure 12. Because the cavities are rather easily compressed by pressure, they positively contribute to the compressibility. ${ }^{39,42,51}$ The significant 
decrease of the compressibility by pressure observed here can be attributed to the decrease of the cavities (compression of the cavities) in these areas or entering water molecule in these cavities.

If the cavities at the $\mathrm{H}$-interface are compressed by the pressure, this may cause friction for the movement of the interface. Because movement of this interface is essential for dissociation, this may subsequently decrease the efficiency of dissociation. If the cavities at the $\mathrm{V}$-interface are compressed, this compression may affect the movement of the C-terminal extension helix ( $\alpha 3$ helix, red helix in Figure 12), which is located around the $\mathrm{V}$-interface and has been reported to be important for the light-induced structural change of BLUF proteins. ${ }^{56-59}$ This C-terminal helix may move upon light excitation. ${ }^{58}$ If such structural change contributes to the dissociation reaction, the compression of the cavities between the monomer units will restrict the conformational change of the C-terminal helix, which may prevent dissociation of the decamer. We believe that compression of cavities between the pentamer rings and within the pentamer ring by pressure are the cause of the decrease of the dissociation reaction yield.

\section{CONCLUSIONS}

The pressure effect on the dissociation reaction of the TePixD decamer was investigated by high-pressure transient grating and high-pressure DLS. It was found that the quantum yield for the dissociation reaction significantly decreased by applying a relatively small pressure. The origin of this pressure effect was investigated by densimetry at high pressures, and it was found that pressure-dependent compressibility occurred. We attribute the origin of the pressure dependence of the reaction yield to be 
the compression of cavities between the monomer units. The present study shows that the fluctuation is the driving force for the dissociation reaction of the TePixD decamer.

\section{ASSOCIATED CONTENT}

\section{Supporting information}

Proof that $D$ of the pentamer is the same as $D$ of the product of the decamer (SI-1), results of cross-linking experiments (SI-2), derivation of eq 8 (SI-3), derivation of eq 9 (SI-4), principle to determine the volume change (SI-5).

These materials are available free of charge via the Internet at http://pubs.acs.org.

\section{AUTHOR INFORMATION}

\section{*Corresponding Author.}

Masahide Terazima

mterazima@kuchem.kyoto-u.ac.jp

tel/FAX: $+81-75-753-4026$

\section{ACKNOWLEDGMENTS}

This work was supported by a Grant-in-aid for Scientific Research on Innovative Areas (research in a proposed research area) (Nos. 20107003 and 25102004) from the Ministry of Education, Culture, Sports, Science and Technology in Japan (to M.T.). 


\section{REFERENCES}

(1) Chalikian, T. V. Volumetric Properties of Proteins. Annu. Rev. Biophys. Biomol. Struct. 2003, 32, 207-235.

(2) Cioni, P.; Gabellieri, E. Protein dynamics and pressure: What can high pressure tell us about protein structural flexibility? Biochim. Biophys. Acta 2011, 1814, 934-941.

(3) Silva, J. L.; Oliveira, A. C.; Vieira, T. C. R. G.; de Oliveira, G. A. P.; Suarez, M. C.; Foguel, D. High-Pressure Chemical Biology and Biotechnology. Chem. Rev. 2014, 114, 7239-7267.

(4) Ohmae, E.; Tatsuta, M.; Abe, F.; Kato, C.; Tanaka, N.; Kunugi, S.; Gekko, K. Effects of pressure on enzyme function of Escherichia coli dihydrofolate reductase. Biochim. Biophys. Acta 2008, 1784, 1115-1121.

(5) Masson, P.; Balny, C. Linear and non-linear pressure dependence of enzyme catalytic parameters. Biochim. Biophys. Acta 2005, 1724, 440-450.

(6) Mozhaev, V. V.; Heremans, K.; Frank, J.; Masson, P.; Balny, C. High Pressure Effects on Protein Structure and Function. Proteins: Struct., Funct., Bioinf 1996, $24,81-91$.

(7) Sato, M.; Ozawa, S.; Ogino, Y. Effects of Pressure on the Sucrose Inversion over an Immobilized Invertase Catalyst. J. Phys. Chem. 1987, 91, 5755-5760.

(8) Taniguchi, Y.; Suzuki, K. Pressure Inactivation of $\alpha$-Chymotrypsin. J. Phys. Chem. 1983, 87, 5185-5193.

(9) Kimura, T.; Sakamoto, K.; Morishima, I.; Ishimori, K. Dehydration in the Folding of Reduced Cytochrome $c$ Revealed by the Electron-Transfer-Triggered Folding under High Pressure. J. Am. Chem. Soc. 2005, 128, 670-671. 
(10) Woenckhaus, J.; Köhling, R.; Thiyagarajan, P.; Littrell, K. C.; Seifert, S.; Royer, C. A.; Winter, R. Pressure-Jump Small-Angle X-Ray Scattering Detected Kinetics of Staphylococcal Nuclease Folding. Biophys. J. 2001, 80, 1518-1523.

(11) Mohana-Borges, R.; Silva, J. L.; Ruiz-Sanz, J.; de Prat-Gay, G. Folding of a pressure-denatured model protein. Proc. Natl. Acad. Sci. U. S. A. 1999, 96, 7888-7893.

(12) Vidugiris, G. J. A.; Markley, J. L.; Royer, C. A. Evidence for a molten globule-like transition state in protein folding from determination of activation volumes. Biochemistry 1995, 34, 4909-4912.

(13) Brindell, M.; Stawoska, I.; Orzeł, Ł.; Łabuz, P.; Stochel, G.; van Eldik, R. Application of high pressure laser flash photolysis in studies on selected hemoprotein reactions. Biochim. Biophys. Acta 2008, 1784, 1481-1492.

(14) Frauenfelder, H.; Alberding, N. A.; Ansari, A.; Braunstein, D.; Cowen, B. R.; Hong, M. K.; Iben, I. E. T.; Johnson, J. B.; Luck, S. Proteins and Pressure. J. Phys. Chem. $1990,94,1024-1037$

(15) Adachi, S.; Morishima, I. The Effects of Pressure on Oxygen and Carbon Monoxide Binding Kinetics for Myoglobin. A high pressure laser flash photolysis study. $J$. Biol. Chem. 1989, 264, 18896-18901.

(16) Moglich, A.; Yang, X.; Ayers, R. A.; Moffat, K. Structure and Function of Plant Photoreceptors. Annu. Rev. Plant Biol. 2010, 61, 21-47.

(17) Kita, A.; Okajima, K.; Morimoto, Y.; Ikeuchi, M.; Miki, K. Structure of a Cyanobacterial BLUF Protein, Tll0078, Containing a Novel FAD-binding Blue Light Sensor Domain. J. Mol. Biol. 2005, 349, 1-9.

(18) Gauden, M.; Yeremenko, S.; Laan, W.; van Stokkum, I. H.; Ihalainen, J. A.; van Grondelle, R.; Hellingwerf, K. J.; Kennis, J. T. Photocycle of the Flavin-binding 
Photoreceptor AppA, a Bacterial Transcriptional Antirepressor of Photosynthesis Genes. Biochemistry 2005, 44, 3653-3662.

(19) Fukushima, Y.; Okajima, K.; Shibata, Y.; Ikeuchi, M.; Itoh, S. Primary Intermediate in the Photocycle of a Blue-Light Sensory BLUF FAD-protein, Tll0078, of Thermosynechococcus elongatus BP-1. Biochemistry 2005, 44, 5149-5158.

(20) Laan, W.; van der Horst, M. A.; van Stokkum, I. H.; Hellingwerf, K. J. Initial Characterization of the Primary Photochemistry of AppA, a Blue-light-using Flavin Adenine Dinucleotide-domain Containing Transcriptional Antirepressor Protein from Rhodobacter sphaeroides:A Key Role for Reversible Intramolecular Proton Transfer from the Flavin Adenine Dinucleotide Chromophore to a Conserved Tyrosine? Photochem. Photobiol. 2003, 78, 290-297.

(21) Okajima, K.; Fukushima, Y.; Suzuki, H.; Kita, A.; Ochiai, Y.; Katayama, M.; Shibata, Y.; Miki, K.; Noguchi, T.; Itoh, S.; Ikeuchi, M. Fate Determination of the Flavin Photoreceptions in the Cyanobacterial Blue Light Receptor TePixD (Tll0078). J. Mol. Biol. 2006, 363, 10-18.

(22) Tanaka, K.; Nakasone, Y.; Okajima, K.; Ikeuchi, M.; Tokutomi, S.; Terazima, M. Oligomeric-State-Dependent Conformational Change of the BLUF Protein TePixD (Tll0078). J. Mol. Biol. 2009, 386, 1290-1300.

(23) Kuroi, K.; Tanaka, K.; Okajima, K.; Ikeuchi, M.; Tokutomi, S.; Terazima, M. Anomalous diffusion of TePixD and identification of the photoreaction product. Photochem. Photobiol. Sci. 2013, 12, 1180-1186.

(24) Kuroi, K.; Okajima, K.; Ikeuchi, M.; Tokutomi, S.; Terazima, M. Transient conformational fluctuation of TePixD during a reaction. Proc. Natl. Acad. Sci. U. S. A. 2014, $41,14764-14769$. 
(25) Okajima, K.; Yoshihara, S.; Fukushima, Y.; Geng, X.; Katayama, M.; Higashi, S.; Watanabe, M.; Sato, S.; Tabata, S.; Shibata, Y.; Itoh, S.; Ikeuchi, M. Biochemical and Functional Characterization of BLUF-Type Flavin-Binding Proteins of Two Species of Cyanobacteria. J. Biochem. (Tokyo) 2005, 137, 741-750.

(26) Hoshihara, Y.; Kimura, Y.; Matsumoto, M.; Nagasawa, M.; Terazima, M. An optical high-pressure cell for transient grating measurements of biological substance with a high reproducibility. Rev. Sci. Instrum. 2008, 79, 034101.

(27) Terazima, M. Photothermal Studies of Photophysical and Photochemical Processes by the Transient Grating Method. In Advances in Photochemistry; John Wiley \& Sons, Inc., 2007; pp 255.

(28) Terazima, M. Molecular volume and enthalpy changes associated with irreversible photo-reactions. J. Photochem. Photobiol., C 2002, 3, 81-108.

(29) Terazima, M. Time-dependent intermolecular interaction during protein reactions. Phys Chem Chem Phys 2011, 13, 16928-16940.

(30) Terazima, M. Diffusion coefficients as a monitor of reaction kinetics of biological molecules. Phys Chem Chem Phys 2006, 8, 545-557.

(31) Gekko, K.; Araga, M.; Kamiyama, T.; Ohmae, E.; Akasaka, K. Pressure dependence of the apparent specific volume of bovine serum albumin: Insight into the difference between isothermal and adiabatic compressibilities. Biophys. Chem. 2009, 144, $67-71$.

(32) Masuya, M. A numerical calculation program of molecular surface area, volume, and solvation energy. http://biocomputing.cc/nsol/ (accessed January 2015).

(33) Voss, N. R.; Gerstein, M. 3V: cavity, channel and cleft volume calculator and extractor. Nucleic Acids Res 2010, 38, W555-W562. 
(34) Tanaka, K.; Nakasone, Y.; Okajima, K.; Ikeuchi, M.; Tokutomi, S.; Terazima, M. A way to sense light intensity: Multiple-excitation of the BLUF photoreceptor TePixD suppresses conformational change. FEBS Lett. 2011, 585, 786-790.

(35) Wonham, J. O. N. Effect of Pressure on the Viscosity of Water. Nature $1967,215,1053-1054$.

(36) Dzwolak, W.; Kato, M.; Taniguchi, Y. Fourier transform infrared spectroscopy in high-pressure studies on proteins. Biochim. Biophys. Acta 2002, 1595, $131-144$

(37) Boonyaratanakornkit, B. B.; Park, C. B.; Clark, D. S. Pressure effects on intra- and intermolecular interactions within proteins. Biochim. Biophys. Acta 2002, 1595, 235-249.

(38) Gekko, K.; Hasegawa, Y. Compressibility-Structure Relationship of Globular Proteins. Biochemistry 1986, 25, 6563-6571.

(39) Kamiyama, T.; Gekko, K. Effect of ligand binding on the flexibility of dihydrofolate reductase as revealed by compressibility. Biochim. Biophys. Acta 2000, 1478, 257-266.

(40) Akasaka, K. Highly Fluctuating Protein Structures Revealed by Variable-Pressure Nuclear Magnetic Resonance. Biochemistry 2003, 42, 10875-10885.

(41) Refaee, M.; Tezuka, T.; Akasaka, K.; Williamson, M. P. Pressure-dependent Changes in the Solution Structure of Hen Egg-white Lysozyme. J. Mol. Biol. 2003, 327, 857-865.

(42) Mimura, S.; Yamato, T.; Kamiyama, T.; Gekko, K. Nonneutral evolution of volume fluctuations in lysozymes revealed by normal-mode analysis of compressibility. Biophys. Chem. 2012, 161, 39-45. 
(43) Roche, J.; Caro, J. A.; Norberto, D. R.; Barthe, P.; Roumestand, C.;

Schlessman, J. L.; Garcia, A. E.; García-Moreno E., B.; Royer, C. A. Cavities determine the pressure unfolding of proteins. Proc. Natl. Acad. Sci. U. S. A. 2012, 109, 6945-6950.

(44) Cooper, A. Thermodynamic fluctuations in protein molecules. Proc. Natl. Acad. Sci. U. S. A. 1976, 73, 2740-2741.

(45) Silva, J. L.; Weber, G. Pressure Stability of Proteins. Annu. Rev. Phys. Chem. 1993, 44, 89-113.

(46) Chalikian, T. V.; Totrov, M.; Abagyan, R.; Breslauer, K. J. The Hydration of Globular Proteins as Derived from Volume and Compressibility Measurements: Cross Correlating Thermodynamic and Structural Data. J. Mol. Biol. 1996, 260, 588-603.

(47) Richmond, Timothy J. Solvent accessible surface area and excluded volume in proteins: Analytical equations for overlapping spheres and implications for the hydrophobic effect. J. Mol. Biol. 1984, 178, 63-89.

(48) Chalikian, T. V. Structural Thermodynamics of Hydration. J. Phys. Chem. $B 2001,105,12566-12578$.

(49) Gerstein, M.; Chothia, C. Packing at the protein-water interface. Proc. Nat1. Acad. Sci. U. S. A. 1996, 93, 10167-10172.

(50) Svergun, D. I.; Richard, S.; Koch, M. H. J.; Sayers, Z.; Kuprin, S.; Zaccai, G. Protein hydration in solution: Experimental observation by $x^{-r a y}$ and neutron scattering. Proc. Nat1. Acad. Sci. U. S. A. 1998, 95, 2267-2272.

(51) Gekko, K. Compressibility gives new insight into protein dynamics and enzyme function. Biochim. Biophys. Acta 2002, 1595, 382-386.

(52) Gekko, K.; Yamagami, K. Compressibility and Volume Changes of Lysozyme due to Inhibitor Binding. Chem. Lett. 1998, 27, 839-840. 
Son, I.; Selvaratnam, R.; Dubins, D. N.; Melacini, G.; Chalikian, T. V.

Ultrasonic and Densimetric Characterization of the Association of Cyclic AMP with the cAMP-Binding Domain of the Exchange Protein EPAC1. J. Phys. Chem. B 2013, 117, 10779-10784.

(54) Gekko, K.; Obu, N.; Li, J.; Lee, J. C. A Linear Correlation between the Energetics of Allosteric Communication and Protein Flexibility in the Escherichia coli Cyclic AMP Receptor Protein Revealed by Mutation-Induced Changes in Compressibility and Amide Hydrogen-Deuterium Exchange. Biochemistry 2004, 43, 3844-3852.

(55) Gekko, K.; Noguchi, H. Compressibility of Globular Proteins in Water at $25^{\circ}$ C. J. Phys. Chem. 1979, 83, 2706-2714.

(56) Masuda, S. Light Detection and Signal Transduction in the BLUF Photoreceptors. Plant Cell Physiol. 2013, 54, 171-179.

(57) Zoltowski, B. D.; Gardner, K. H. Tripping the Light Fantastic: Blue-Light Photoreceptors as Examples of Environmentally Modulated Protein-Protein Interactions. Biochemistry 2010, 50, 4-16.

(58) Wu, Q.; Gardner, K. H. Structure and Insight into Blue Light-Induced Changes in the BlrP1 BLUF Domain. Biochemistry 2009, 48, 2620-2629.

(59) Barends, T. R.; Hartmann, E.; Griese, J. J.; Beitlich, T.; Kirienko, N. V.; Ryjenkov, D. A.; Reinstein, J.; Shoeman, R. L.; Gomelsky, M.; Schlichting, I. Structure and mechanism of a bacterial light-regulated cyclic nucleotide phosphodiesterase. Nature 2009, 459, 1015-1018. 
Table of Contents

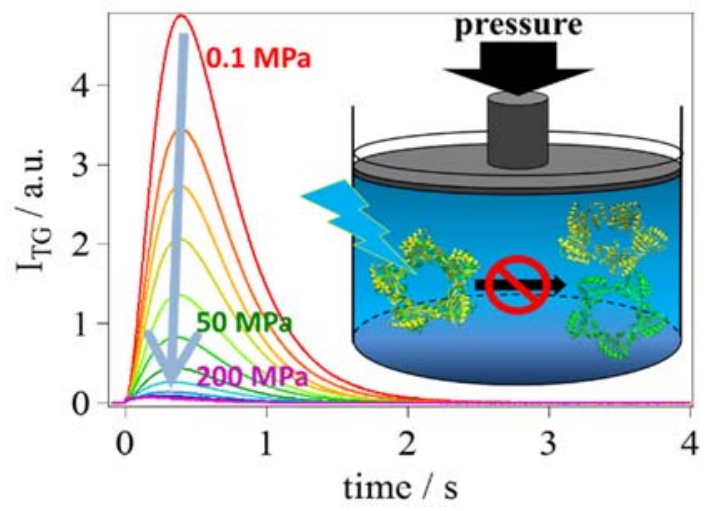

